\title{
The RESIN Climate Change Adaptation Project and its Simple Modeling Approach for Risk-oriented Vulnerability Assessment
}

\author{
Daniel Lückerath, Manfred Bogen, Erich Rome, Betim Sojeva, \\ Oliver Ullrich, Rainer Worst, Jingquan Xie
}

Fraunhofer Institute for Intelligent Analysis and Information Systems IAIS

Schloss Birlinghoven, 53757 Sankt Augustin, Germany; *daniel.lueckenrath@iais.fraunhofer.de

SNE 28(2), 2018, 49 - 54, DOI: 10.11128/sne.28.pn. 10412

Received: April 10, 2018 (Selected ASIM GMMS/STS 2018

Postconf. Publ.), Accepted: May 15, 2018

SNE - Simulation Notes Europe, ARGESIM Publisher Vienna,

ISSN Print 2305-9974, Online 2306-0271, www.sne-journal.org

\begin{abstract}
Urban population centers are especially vulnerable to extreme weather events and climate change. Local decision makers demand standardized processes, methods, and tools enabling them to design and implement climate adaptation strategies for their specific contexts. The project "Climate Resilient Cities and Infrastructures - RESIN" aims at providing such a set of methods and tools; it utilizes the impact chain modeling approach to capture and represent cause-effect relationships underlying risks and vulnerabilities in urban population centers, enabling a systematic analysis and evaluation. Highlighting on a number of concepts, such as hazard, exposure, stressors, coping capacity, and vulnerability, these impact chains constitute the base for further quantitative modeling steps.
\end{abstract}

\section{Introduction}

High concentrations of residents and economic assets render urban population centers especially vulnerable to the impact of extreme weather events and consequences of climate change (see [1]). Such disasters endanger large numbers of residents and critical infrastructure systems at the same time, thereby also impacting inter-regional and global economic networks (see [2]). The trend towards increasing urbanization in Europe - by $205082 \%$ of all Europeans are expected to live in urban population centers (see [3]) - and the increasing dependencies of infrastructure components make it necessary for municipalities to develop proactive strategies to increase their resilience against climate- related disasters.

Climate change adaptation measures, in contrast to climate protection efforts that are mainly aimed at reducing greenhouse gas emissions, are designed to reduce the impact of climate change on social and biological systems, such as urban population centers. Unfortunately, not many standardized methods and toolsets exist today that enable municipal decision makers to plan, assess, and implement adaptation measures, thereby helping them to consider, analyze, and evaluate risks and vulnerabilities under specific, climate change related scenarios. In the context of such a systematic scenario analysis, the application of a simulation model can be of considerable benefit. The foundation of such a model - and by extension of effective adaptation measures - is a comprehensive understanding of the risks and vulnerabilities themselves.

This paper describes characteristics and generation of impact chains representing cause-effect relationships that form risks and vulnerabilities of urban population centers in the context of the ongoing EU project "Climate Resilient Cities and Infrastructures - RESIN" (see [6]). The project is aimed at developing practical and applicable methods and tools to support municipalities in designing and implementing climate adaptation strategies for their local contexts. RESIN also systematically compares and evaluates methods for climate change adaptation in order to move towards a formal standardization of adaptation strategies.

RESIN is one of several interdisciplinary, practicebased research projects investigating climate resilience in European cities. 
The EU project "Reconciling Adaptation, Mitigation and Sustainable development for citiES - RAMSES" (see [4]) that was concluded recently, developed methods and tools to quantify evidence of the impacts of climate change and the costs and benefits of adaptation measures to cities. The EU project "Smart Mature Resilience - SMR" (see [5]) aims at developing a resilience management guideline to support city decision-makers in developing and implementing resilience measures.

The paper continues with a short introduction to the RESIN project and its fundamental process (see Section 1). It then goes on to describe the impact chain modeling approach used to capture and represent causeeffect relationships underlying risks and vulnerabilities in urban population centers, enabling a systematic analysis and evaluation (see Section 2). The paper concludes with a short description of the steps necessary to complete the modeling process based on the generated impact chains (see Section 3).

\section{The RESIN Project}

RESIN investigates climate change adaptation practices in European cities in order to develop standardized methods and decision support tools that decision makers can use to develop local adaptation strategies.
The project builds on previous research by combining existing approaches to climate change adaptation and disaster risk management while taking into account all of the core elements of the urban system and their interrelations. One of the central aims of RESIN is to provide standardized methods and tools for comprehensive risk-oriented vulnerability assessments of an urban population center. These efforts are based on the latest state of discussion and conceptual approaches of the Intergovernmental Panel on Climate Change (IPCC) Assessment Report 5 (AR5, see [7]). RESIN views a city as a "system of systems" comprised of complex social, ecological, and technical sub-systems that overlap and interact with one another.

All RESIN methods and tools are being developed by means of co-creation with the cities of Bilbao (Spain), Greater Manchester (United Kingdom), Paris (France), and Bratislava (Slovakia). The first cocreation process with Bilbao, aiming at producing a vulnerability and risk mapping on a neighborhood (barrios) scale, started in July 2016 and concluded in September 2017.

Process, methods, and tools are standardized and can be applied to more European urban population centers, but at the same time support the tailoring to the specific needs of a municipality, depending on the varying degrees of maturity of their adaptation processes.

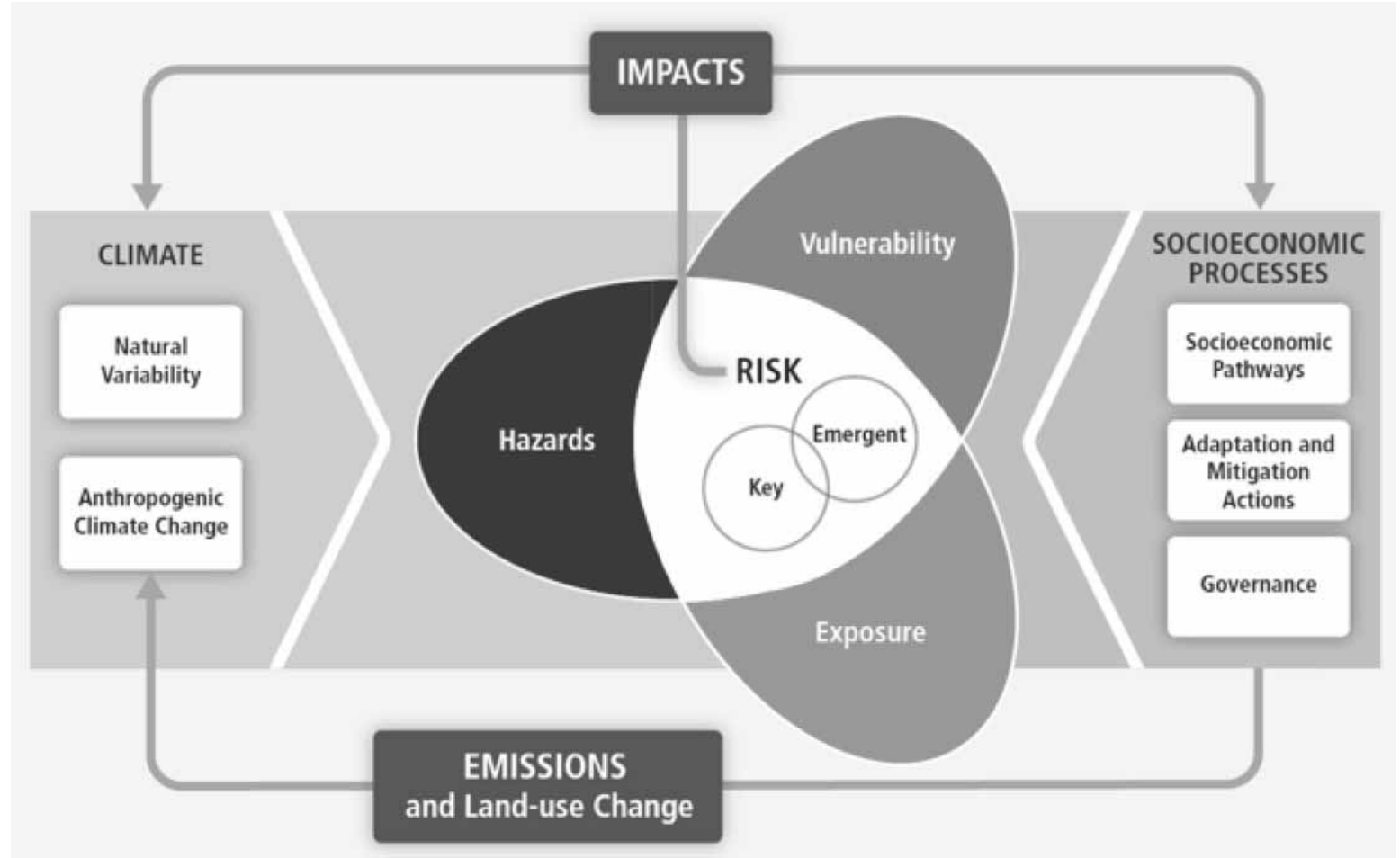

Figure 1. Risks as compositions of hazards, exposure, and vulnerability (source: [7]). 


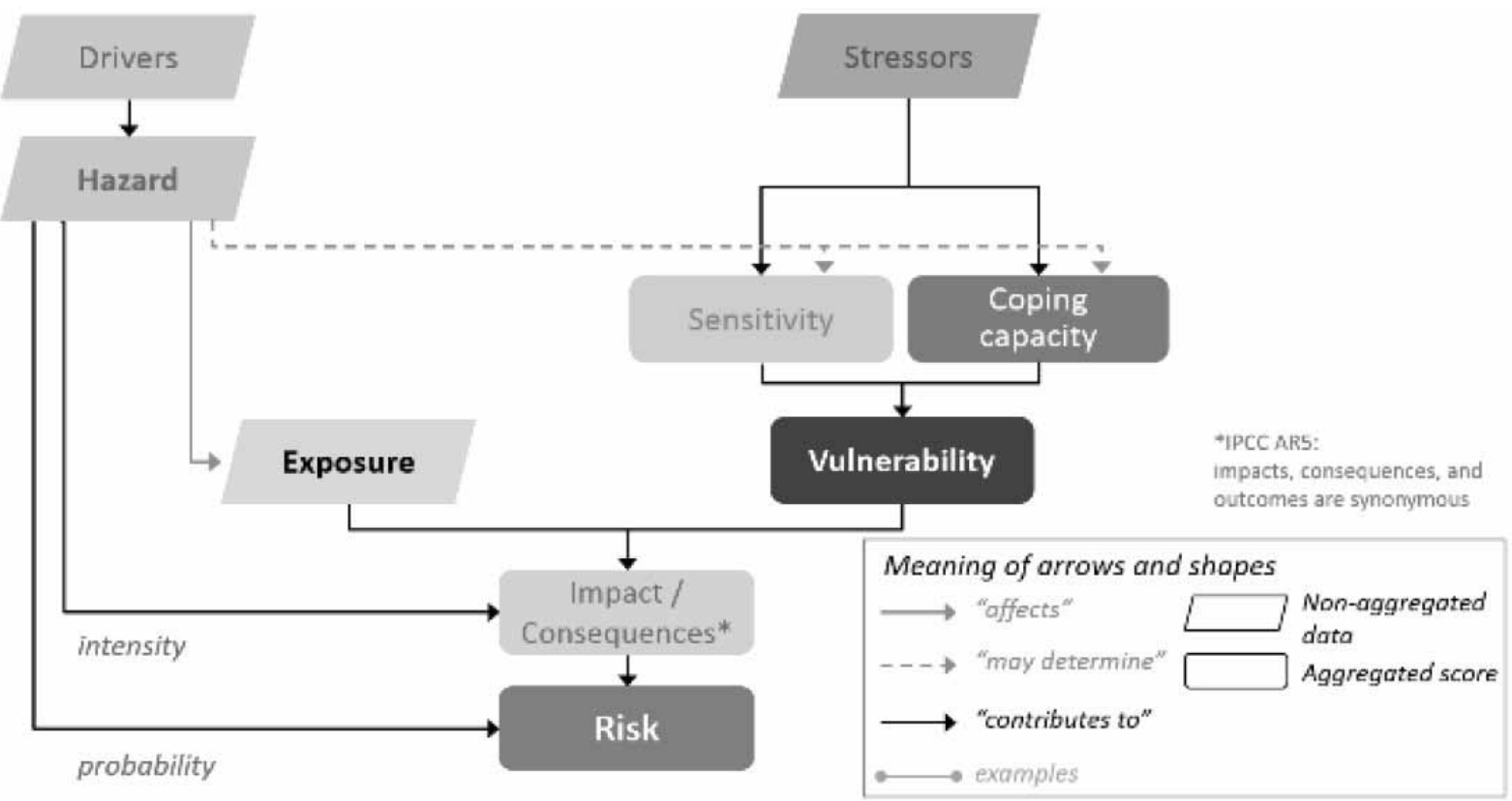

Figure 2. A risk-oriented vulnerability assessment schema (source: [11]).

The application of the RESIN conceptual framework follows four principal stages (see [8]):

1. Assessing climate risks

2. Developing adaptation objectives

3. Prioritizing adaptation options

4. Developing an implementation plan

The impact chain modeling technique described in the following section is part of the modelling process during the first stage of this adaptation planning process.

In RESIN, the design chosen for the vulnerability assessment process is based on the indicator-based framework provided by "The Vulnerability Sourcebook" (see [9]). Since the sourcebook is based on IPCC AR4 (see [10]), the framework had to be adapted to account for the conceptual move to a risk-based approach by IPCC AR5 (see [7]). Compared to AR4, AR5 defines and uses terms like vulnerability and exposure in different ways: Where exposure to climate change hazards was once considered to be part of vulnerability - alongside sensitivity to hazards and capacity to adapt -, the move to risk has separated out exposure.

As a result, risk is now regarded by the IPCC as a function of climate hazard, exposure, and vulnerability (see Figure 1).

\section{Using Impact Chains to Model Risks and Vulnerabilities}

Impact chains are tools (described in [9]) for capturing and structuring the components of a particular causeeffect relationship. They describe the basic connections between the elements, and prepare the selection of quantitative indicators for many of the components. Experiences during the co-creation processes with local experts from Bilbao, Bratislava, Manchester, and Paris show that impact chain diagrams are easy to grasp and apply, and that participants appreciate the structured modeling approach. As a result, impact chains are not exhaustive, but describe the common understanding of the stakeholders present at the workshops. Often, experts found that impact chain diagrams gave them first clues towards potential adaptation measures.

As part of impact chain modeling, RESIN utilizes a number of concepts (see Figure 2) to derive overall risk estimations: drivers, hazard, exposure, stressors, sensitivity, coping capacity, vulnerability, and impacts.

A hazard is defined as "...the potential occurrence of a natural or human-induced physical event or trend, or physical impact that may cause loss of life, injury, or other health impacts, as well as damage and loss to property, infrastructure, livelihoods, service provision, and environmental resources" (see [7]). 
A climate-related hazard is a special case that is (at least partially) caused by climatic drivers. Examples include flooding, heatwave, drought, and water scarcity.

Exposure refers to the objects or systems that are exposed: The presence of people, livelihoods, species or ecosystems, environmental services and resources, infrastructure, or economic, social, or cultural assets in specific places that could be adversely affected.

Non-climatic trends and events, which are called stressors, can have an important effect on an exposed system. Examples are population growth or change of land-use; a larger percentage of sealed surface will in general increase the susceptibility to flooding events and thus the vulnerability of all exposed objects.

Different objects are more or less sensitive to a hazard. This is captured by the concept of sensitivity, defined as the degree to which an exposed object, species or system could be affected by the considered hazard. As such, sensitivity towards a hazard can be perceived as a property of an exposed object in regard to a specific hazard. Examples for sensitivity include the degrees of surface sealing, age and density of a population, household-income, or elevation and density of buildings.

Coping capacity is defined as "the ability of people, institutions, organizations, and systems, using available skills, values, beliefs, resources, and opportunities, to address, manage, and overcome adverse conditions in the short to medium term" (see [12]). Examples include the draining capacity of sewer systems, a dike's height, education and awareness of the population, and availability of early warning systems, while examples for adaptive capacity include diversity of economic activities, state of the city infrastructure, network redundancy, diversity of land-use, or availability of hospital beds.

Vulnerability is derived from the interplay of stressors, sensitivity, and coping capacity. It contributes directly to the impact or consequences that a hazard causes to the exposed objects.

Risk is classically computed by multiplying the probability of an adverse event with the magnitude of the expected consequences (see [12]). A risk assessment takes into account the characteristics and intensity of the considered hazard, as well as the set of objects exposed to it. The probability of a hazard affecting the set of objects may be estimated from extrapolating historical data or simulation results concerning the frequency of the hazard and the development of the objects. The vulnerability of the objects exposed to the hazard then determines the consequences.

The RESIN vulnerability assessment process starts with a systematic analysis and selection of hazards, drivers, and stressors relevant to the urban area under examination. These results serve as a base for the detailed planning of the assessment and ensures that the usually limited - resources available for the assessment are spent on the most pressing current and future hazards, and no other threats or possible dependencies between different hazards are overlooked. In addition, a thorough documentation of the rationale for selecting hazards, drivers, and stressors is recommended to ensure that future assessments or re-evaluations yield comparable results.

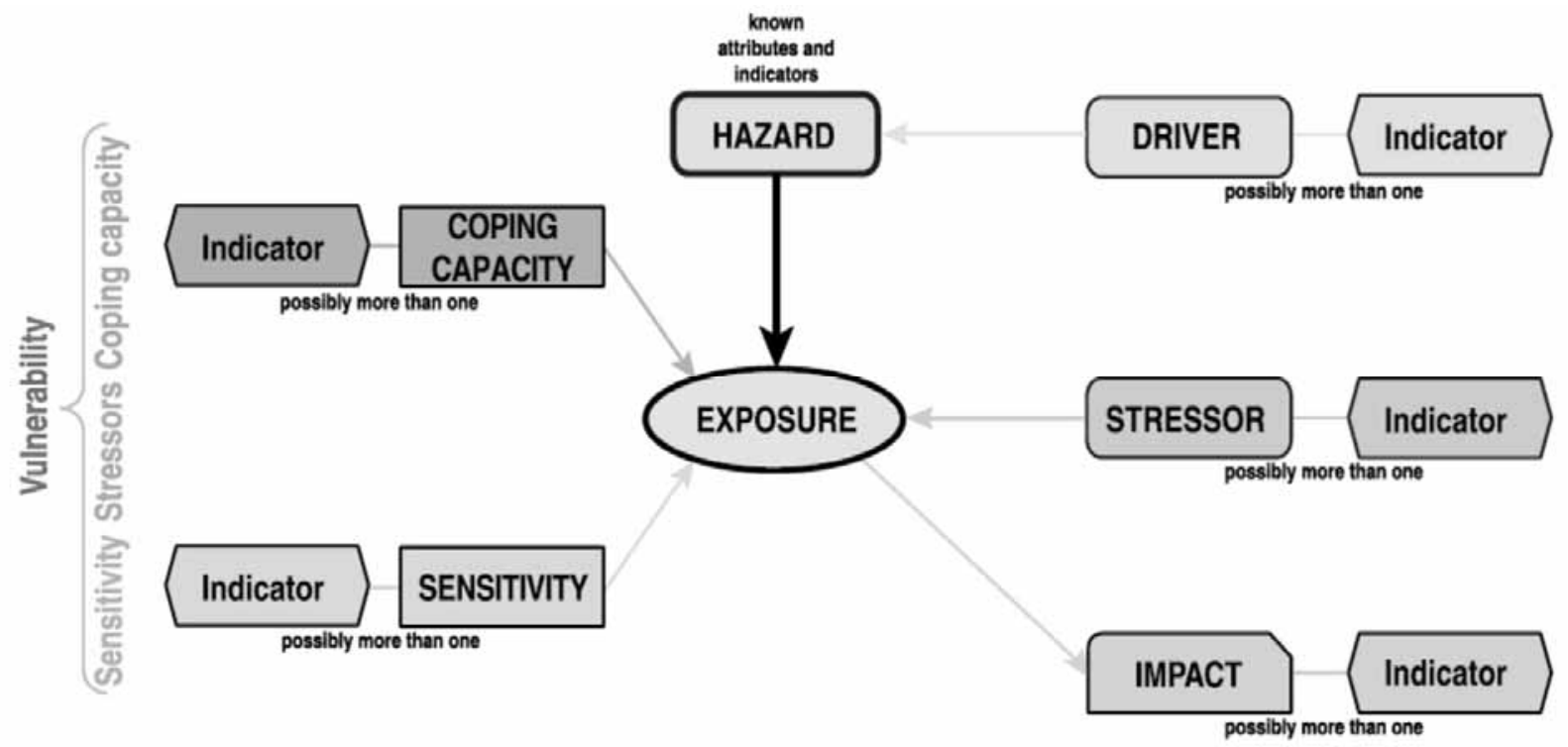

Figure 3. Generic impact chain diagram schema (source: [11]). 
Once the vulnerability assessment is completed, impact chain diagrams (see Figure 3) are developed. The diagrams then visually illustrate cause-effect relationships between the elements contributing to the impact of a given combination of hazards and exposed objects.

RESIN impact chain diagrams explicitly differentiate between hazards on the one hand and impacts and consequences on the other (see Figure 4) - thus representing cause and effect. In addition, the diagrams model relationships between sensitivity and coping capacity, and exposed object. Each element of an impact chain may be described in a qualitative way by specifying attributes, such as "green infrastructure" for coping capacity, and later also in a quantitative way by assigning measurable indicators, such as "percentage of green area per city district". Usually, impact chain diagrams are developed during collaborative workshops with experts and stakeholders.

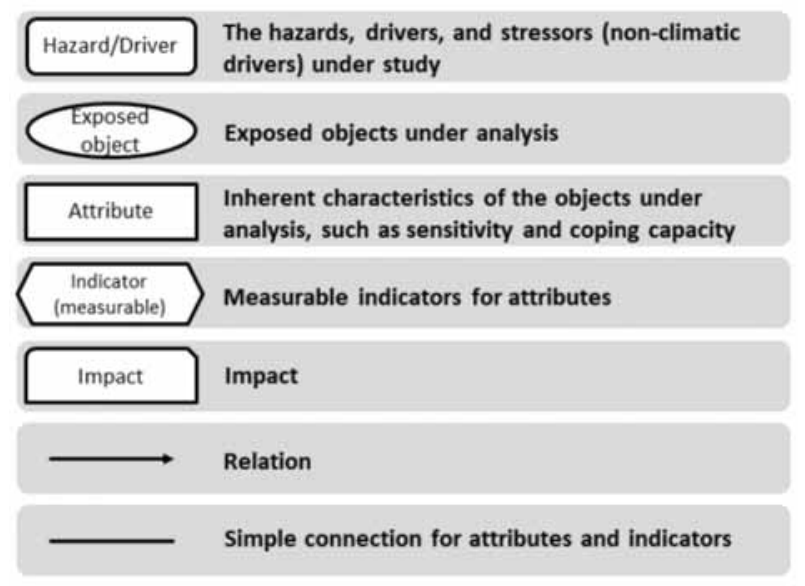

Figure 4. Elements used in impact chain diagrams (source: [11]).

Figure 5, developed during a co-creation workshop with local experts, shows an example impact chain for the hazard-exposure combination heatwave on public health for the city of Bilbao. Here, a heatwave is defined as a day when the average temperature exceeds $32^{\circ} \mathrm{C}$. Both the coping capacity and the sensitivity indicators contain a mixture of infrastructure related measures as well as social indicators, such as the amount of green infrastructure and the percentage of elderly people. On the right side of the diagram, the impact indicators cover mainly health related and economic consequences.
The impact chain development (without the definition of measureable indicators) concludes the qualitative part of the assessment process and is a very valuable outcome itself. End-users without the necessary resources - both in terms of personnel and knowledge may decide to end the vulnerability assessment at this point. Others may opt to go for the quantitative part outlined below.

\section{Further Steps}

Based on the insights gained during this phase, impact chains are annotated by further identifying measureable indicators for all identified elements, and by gathering necessary data for their calculation. To ease the indicator selection process, established directories of standard indicators can be employed. Such directories can be found, for example, in the annex of the Vulnerability Sourcebook (see [9]), the annex of the Covenant of Mayors for Climate and Energy Reporting Guidelines (see [13]) or the indicator database of the European Union Framework Programme 7 project MOVE (Methods for the Improvement of Vulnerability Assessment in Europe, see [14]). These annotated impact chains are then utilized as a base for evaluation and simulation models of impacts, hazards, sensitivity, coping capacity, or stressors - which in turn are applied to move from risk assessments based on historic data to risk assessment of future (simulated) scenarios.

\section{Acknowledgments}

The authors thank their partners in the RESIN consortium for their valuable contributions during the development and test process. This paper is based in part upon work in the framework of the European project "Climate Resilient Cities and Infrastructures - RESIN". This project has received funding from the European Union's Horizon 2020 research and innovation programme under grant agreement no. 653522.

The sole responsibility for the content of this publication lies with the authors. It does not necessarily represent the opinion of the European Union. Neither the EASME nor the European Commission are responsible for any use that may be made of the information contained therein. 


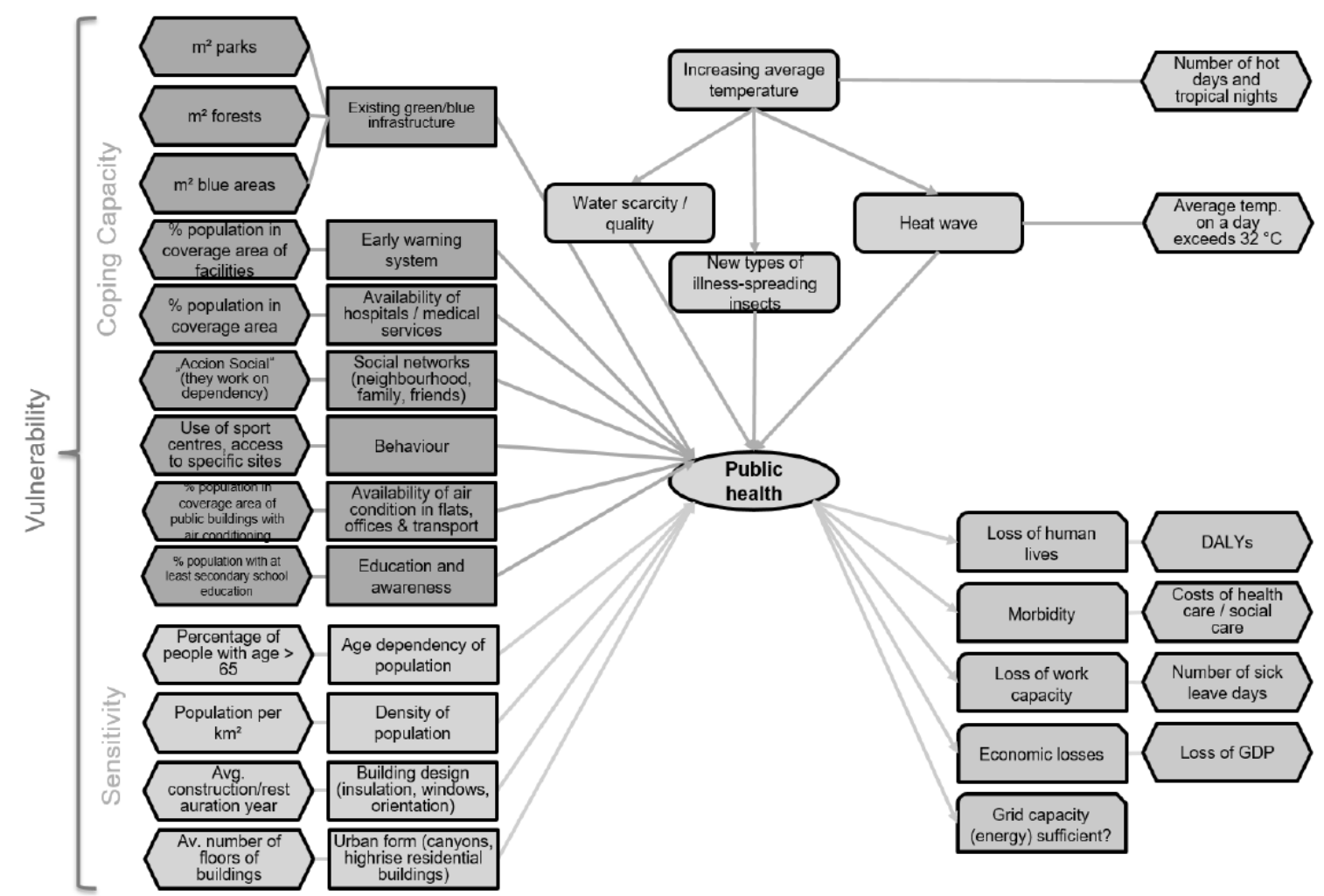

Figure 5. Example impact chain for the hazard-exposure combination heat wave on public health in the city of Bilbao (source:[11]).

\section{References}

[1] Coletti A, De Nicola A, Villani ML. Building climate change into risk assessment. In: Natural Hazards, Vol. 84, Issue 2, pp. 1307-1325, 2016.

[2] Burke M, Hsiang SM, Miguel E. Global non-linear effects of temperature on economic production. In: Nature, Vol. 527, pp. 235-239, 2015.

[3] United Nations, Department of Economic and Social Affairs, Population Division. World Urbanization Prospects: The 2014 Revision, Highlights. United Nations, 2014.

[4] RAMSES - Reconciling adaptation, mitigation and sustainable development for cities. URL, visited on Dec 05, 2017, http://www.ramsescities.eu/

[5] SMR - Smart Mature Resilience. URL, visited on Dec 05, 2017. http://smr-project.eu

[6] RESIN - Climate Resilient Cities and Infrastructures. URL, visited on Nov 11, 2017: http://www.resincities.eu/

[7] Intergovernmental Panel on Climate Change (IPCC). Summary for Policymakers, in: Climate Change 2014: Impacts, Adaptation, and Vulnerability. Part A: Global and Sectoral Aspects. Cambridge University Press, Cambridge, United Kingdom, and New York, NY, USA, pp. 1-32, 2014.
[8] Carter J, Connelly A, Handley J, Hincks S, Karvonen A. Deliverable D1.3 Conceptual Framework. EU H2020 Project RESIN, Manchester, UK, 2016.

[9] German Federal Ministry for Economic Cooperation and Development. The Vulnerability Sourcebook. Concept and guidelines for standardised vulnerability assessments. Deutsche Gesellschaft für Internationale Zusammenarbeit, Bonn and Eschborn, Germany, 2014.

[10] IPCC. Climate Change 2007: Synthesis Report. Pachauri, R.K and Reisinger, A. (eds.) IPCC, Geneva, Switzerland, 2007.

[11] Rome E, Bogen M, Worst R, Lückerath D, Sojeva B, Voss H, Voss N, Xie J. Deliverable D2.3 Realisation and implementation IVAVIA. EU H2020 Project RESIN, Sankt Augustin, Germany, 2017.

[12] IPCC. Annex II: Glossary, in: Climate Change: Synthesis Report. R.K. Pachauri and L.A. Meyer (eds.) IPCC, Geneva, Switzerland, pp. 117-130, 2014.

[13] Neves A, Blondel L, Brand K, Hendel Blackford S, Rivas Calvete S, Iancu A, Melica G, Koffi Lefeivre B, Zancanella P, Kona A. The Covenant of Mayors for Climate and Energy Reporting Guidelines. EUR $28160 \mathrm{EN}$; doi:10.2790/586693, 2016.

[14] MOVE Indicator Database Visualiser of the FP7 project MOVE. URL, visited on Oct 25, 2017: http://www.gi4drr.org/move/ 